\title{
Request - Response Based Power Aware Routing Protocol for Wireless Heterogeneous Sensor Networks
}

\author{
M. Viju Prakash ${ }^{1, *}$ and B. Paramasivan ${ }^{2}$ \\ 1, *Assistant Professor, Department of Computer Science and Engineering, \\ St. Xavier's Catholic College of Engineering, Nagercoil, India \\ vijuprakash@sxcce.edu.in \\ ${ }^{2}$ Professor and Head, Department of Computer Science and Engineering, \\ National Engineering College, Kovilpatti, India \\ bparamasivan@yahoo.co.in
}

\begin{abstract}
There are different applications in wireless sensor networks, where sensors with different capacities are deployed. So designing a reliable and scalable routing protocol for a real world wireless heterogeneous sensor network is an interesting task. In this paper, we propose a novel power aware routing for wireless heterogeneous sensor network, which can provide loop less, stateless routing without using any prior neighborhood information. It utilizes both symmetric and asymmetric links to forward a data from source to sink. This protocol explores the neighbor node relations and chooses the best-hop based on node power. It eliminates the low-powered nodes to become hopnodes in the contention process. It also prolongs the network lifetime. Analysis shows that this scheme significantly outperforms the existing protocol in wireless heterogeneous sensor networks.
\end{abstract}

Keywords: Heterogeneous, Power-aware routing, Response, Request, Reverse path, Wireless sensor networks

\section{Introduction}

Recent advances in electronics have paved the way for developing RFID enabled lowcost chip called wireless sensor networks (WSNs). WSNs are widely used in military and civilian applications such as target tracking [1], sensor based automated home [2], weather and environment monitoring [3] and disaster rescuing [4]. These sensors collect data related to pressure, humidity, temperature and light etc. from the environment, process them and forward it to hop-nodes for further processing. These sensor nodes may not have the same sensing power and transmission range. This irregularity in nodes creates asymmetric links in between wireless heterogeneous sensor networks (WHSNs).

After data collection in node level, one major issue in heterogeneous sensor networks is how to route data to the destination (mostly it is sink) efficiently [5 - 7]. Since these sensors have dissimilar transmission ranges, there will be asymmetric links in the communication graph i.e. if node $u$ can reach node $v$, but node $v$ cannot reach node $u$. It formulates an asymmetric link between $u$ and $v$. Thus the off-the-shelf routing protocols for WSNs are not appropriate or work with higher overhead [8] to WHSNs. So WHSN routing protocols need to be changed and should have the following requirements: (1) Reliable and loop-free path identification based on node residual energy; (2) Use recent restructured information for scalability.

In this paper, we propose a power aware routing protocol which uses beacon messages to identify asymmetric links between source and neighbor nodes. Then the best-hop is elected from the number of neighbor nodes. This protocol has two parts: the handshake 
part which includes identifying neighbor node relationship and identifying the reverse path if any asymmetric link exists between a source and neighbor node, and the selection part which selects the best-hop based on node power.

One of the chief issues is hot-spot which is not reflected in this work since the main objective is identifying best-hop node based on individual node power in the survival of asymmetric links. Various researches have been lengthily done regarding the hot-spot problems in WSNs [9-12]. So we are addressing the issues abandoned by them.

The rest of the paper is organized as follows: Section II focuses on the related work which gives the detailed survey about routing strategies in both homogeneous and heterogeneous sensor networks. Section III describes preliminaries and system model. Section IV gives the explanation of proposed protocol. Section V deals with the simulations and evaluates the performance of the proposed protocol. Finally the conclusion and future work is drawn in Section VI.

\section{Related Work}

In this section, we give an overview of existing routing protocols in both wireless sensor networks and wireless heterogeneous sensor networks.

\subsection{Routing in Wireless Homogeneous Sensor Networks}

In homogeneous sensor networks, the main assumption is all sensors have the same capabilities in terms of communication, energy, computation, reliability etc. Stojmenovic and Lin et al. [13] described three different fully localized algorithms to minimize energy consumption. Maximizing network lifetime is intended in [14]. Energy efficient beaconless geographic forwarding [15] is an energy efficient node data forwarding scheme which uses the idea of optimum relay search region to identify the best-hop node. The MFR protocol proposed by Takagi and Kleinrock et al. [16] is the earliest geographic algorithm in which each node selects its forwarder which has maximum progress. In [17], $W u$ and Candan et al. proposed a protocol called GPER was proposed for power-efficient routing. Packet Reception Rate (PRR) and transmission distance (DIST) is considered based on realistic physical layer model and PRR X DIST taken as a decision metric in [18]. Heissenbuttel et al. proposed a protocol called Beaconless Routing (BLR) [19] and it uses the idea called Dynamic Forwarding Delay (DFD). Fußler et al. proposed a method called active selection method in the approach called Contention-based forwarding for mobile ad-hoc networks [20] which use some control messages to identify the forwarding nodes. The implicit geographical forwarding (IGF) proposed by Blum et al. [21] and his idea is integrating beaconless routing with IEEE 802.11 MAC layer. However most of the geographic routing schemes work on the basis of hop-count, which is not efficient in terms of power awareness.

Most of the routing protocols use greedy forwarding, but it struggles when a node cannot find a better neighbor than itself. This situation causes local minimum. To recover from a local minimum, few protocols like GFG [22], GPSR [23] and GOAFR+ [24] use planer sub-graph when a local minimum is encountered. Another significant aspect in WSN is called guaranteed data delivery. To provide guaranteed data delivery, most geographic routing algorithms [25-27] select greedy forwarding mode and recovery mode depending on the network topology. However, in applications such as abovementioned, heterogeneous sensors with different capabilities are deployed. So routing protocols of WSNs may be inappropriate to WHSNs because it will not take advantage of the diversity of the sensors.

\subsection{Routing in Wireless Heterogeneous Sensor Networks}

In the literature, few routing protocols are suggested for WHSNs [28-32] where the deployed sensor nodes are separated into powerful and less powerful ones. Powerful 
nodes are considered as cluster head in a group and less powerful nodes become data collection centers. These approaches make a two-tier design of a single protocol: The intra-cluster protocol is used in between data centers and cluster heads. Inter-cluster protocol is used to transfer the data from cluster head to the sink. However in the above mentioned protocols, the capabilities of individual sensors are not fully explored and asymmetric links are not fully utilized. In [33] Xiao Chen et al. proposed ProHet which uses symmetric and asymmetric links in sensor networks and achieves high delivery rate based on probabilistic calculation. It explores the relationship among neighboring nodes whereas it is missing in [34]. However ProHet does not consider individual node power as it is an important issue in heterogeneity.

In this paper we deliberate node power and asymmetric communication links originated from heterogeneity. Our protocol is fully based on individual node power and assures loop-free, stable and power aware routing in wireless heterogeneous sensor networks. It also utilizes a minimum memory in a source node to find the best-hop.

\section{Preliminaries}

\subsection{Definition of Neighbor Relationships in Wireless Heterogeneous Sensor Network}
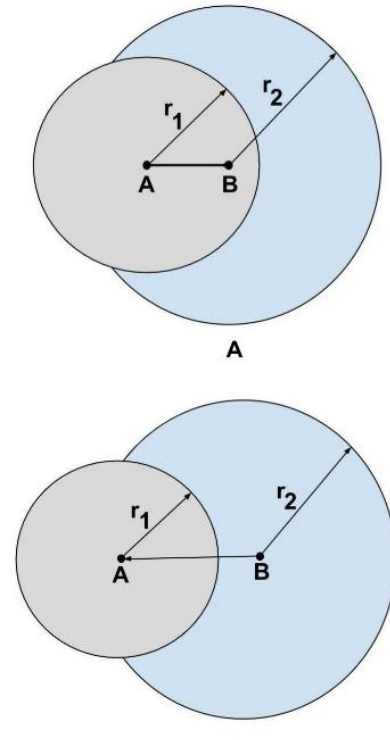

C
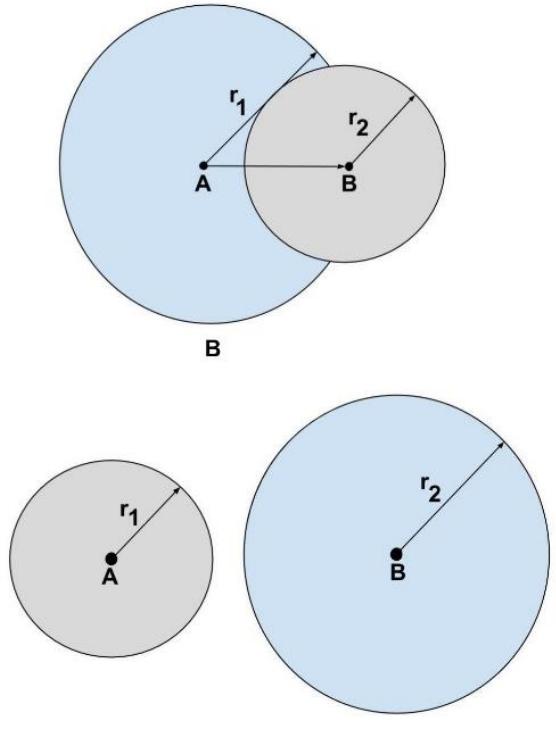

D

Figure 1. Four Different Relationships among Nodes in Heterogeneous Sensor Network

A WHSN can be defined by a directed graph $G=\{V, E\}$, where $V$ is a set of sensor nodes and $E$ is a set of links in the network. There will be four different relationships in the heterogeneous sensor network: (1) In-out neighbor; (2) In-neighbor; (3) Out-neighbor; and (4) Non-neighbor. For example, let us consider two nodes $A$ and $B$, as shown in Figure 1(a), if $A \rightarrow B$ and $B \rightarrow A$ then $A$ and $B$ are in-out neighbor to each other even though $A$ is having radius $r_{1}$ and $\mathrm{B}$ is having radius $r_{2}$. On the other hand as shown in Figure 1(d), if neither $A \rightarrow B$ nor $B \rightarrow A$, they are non-neighbors of each other. Figure 1(b) shows the relationship of in-neighbor of $B$ from $A$ and out-neighbor of $A$ from $B$. As per Figure 1(c), $B$ is an in-neighbor of $A$ and $A$ is out-neighbor of $B$. 


\subsection{Definition of One-Hop and Two-Hop Receiver Model and Limitations of other Models}

A one-hop receiver of a node is either the in-out neighbor or out neighbor of the node. A two-hop receiver is the one-hop neighbor of the one-hop receiver. A two-hop receiver model is also attractive because only local collected information is needed. Here we used both one-hop receiver model in our proposed system and two-hop receiver model in ProHet. When we overlook all the practical aspects, $n$-hop $(n \geq 1)$ neighborhood model can be used. However, the one-hop receiver model is more similar like flooding which can cause more redundant packets. If $n \geq 3$, then neighborhood model introduces more communication overhead because of excessive memory utilization in each node.

\subsection{Network Model}

In this work, we have anticipated that no two nodes locate at the same position. Also it is assumed that every node that has heterogeneous radio transmission has varying transmission ranges $r_{1}, r_{2}, r_{3} \ldots r_{n}$. Each node knows its own location as well as the location of the sink. In this model, Unit Disk Graph (UDG) communication method is employed. As per UDG, any two nodes $u_{1}$ and $u_{2}$ can communicate with each other only if $\left|u_{1} u_{2}\right| \leq r_{1} \cap r_{2}$, where $\left|u_{1} u_{2}\right|$ is the euclidean distance between $u_{1}$ and $u_{2}$.

\subsection{Energy Expenditure Model}

The first order radio model [35] is widely used for evaluating energy consumption in homogeneous sensor networks. We used the modified first order radio model to evaluate the energy consumption of our work. As per first order radio model, the total energy spent for transmitting 1-bit data is the sum of energy spent by a transmitter node and the receiver node. The required energy for transmitting 1-bit data over distance $d$ is $\xi_{\text {transmit }}(d)$ $=x_{11}+x_{2} d^{k}$, where $x_{11}$ is the total energy spent by the transmitter node, $x_{2}$ is the amplification process done at source end and $k$ is the propagation loss exponent. In the receiver side, the required energy for receiving 1-bit data is $\xi_{\text {receive }}(d)=x_{12}$, where $x_{12}$ is the energy spent by the receiver node. Therefore, the total energy consumed by 1-bit to travel from the transmitter to receiver over distance $d$ is

$$
\xi_{\text {total }}(d)=x_{11}+x_{2} d^{k}+x_{12} \equiv x_{1}+x_{2} d^{k}
$$

Where $x_{1}=x_{11}+x_{12}$.

In this work, we considered the energy consumption of intermittent nodes along with the parameters specified in first order radio model. Because of its heterogenic nature, few intermittent nodes may require data transmission from the source node to hop-node. Hence, Equation (1) can be modified as follows.

$$
\xi_{\text {total }}(d)=\xi_{\text {transmit }}(d)+\xi_{\text {receive }}(d)+\xi_{\text {intermittent }}(d)
$$

$\xi_{\text {intermittent }}(d)$ is the total energy spent by the number of intermittent nodes. Let us denote $\xi_{\text {intermittent }}(d)=x_{3}$ and elaborate Equation (2) as follows.

$$
\xi_{\text {total }}(d)=x_{1}+x_{2} d^{k}+x_{3}
$$

\section{The Proposed Protocol}

In this section, we present our proposed protocol, which has two major parts: the handshake part which includes identifying hop-nodes power and a reverse path for the nodes which is having an asymmetric link, the selection part identifies the best-hop node based on node residual power. The details are as follows: 


\subsection{Handshake Part}

If one source node wants to forward a data to the sink and the sink is not in its transmission range, then the source node has to forward the data to hop-node. So, the source node will broadcast a request message. Each neighbor node which receives a request message from a source will store the source ID in its in-neighbor list initially and broadcast its own power and receiver ID as response then waits for an acknowledgement. If the source node $A$ receives a response message from receiver $B$, then the source node will update the neighbor-node ID in its in-out neighbor list along with its node power and will give an acknowledgement to receiver $B$. If an acknowledgement message is received by node $B$, then the node migrates the source node ID from in-neighbor list to in-out neighbor list. Otherwise node $B$ will construct a reverse path to reach node $A$. This acknowledgement is used to identify the neighbor node relationship. Identifying neighbor nodes is explained in Algorithm 1.

\section{Algorithm 1: Identifying Neighbor Nodes and Power}

1. Source node $A$ broadcasts "request" message to all neighbor-nodes.

2. If neighbor node $B$ receives a "request" message from $A$, it records Source ID in the in-neighbor list, and broadcast its power and Receiver ID as "response" and waits for an "acknowledgement" from source $A$.

3. If source node $A$ receives "response" message from $B$, it stores node- $B$ ID in its inout neighbor list and its power. Then, it produces an "acknowledgement" message to the neighbor node $B$.

4. If the neighbor receives an "acknowledgement" from the source, it knows that the broadcasted messages are stored by the source node $A$. So node $B$ will move the source ID from in-neighbor list to in-out neighbor list.

5. If the receiver $B$ does not receive any "acknowledgement" from the source node $A$, then it knows that it is an in-neighbor to the source node $A$. So node $B$ will find a reverse path to reach source node $A$.

Next, for each node that has an in-neighbor, it is necessary to find a reverse path using "find" messages. This method is explained in identifying a reverse path algorithm. This algorithm completely utilizes the asymmetric links of WHSNs. A theoretical study [36] analyzes the asymmetric links in WHSNs which reports that up-to $97 \%$ connectivity can be made successful, if the reverse path length is set to be equal to 3 . In order to restrict the reverse path length, we used expiration bits and its size is 3 .

\section{Algorithm 2: Identifying a Reverse Path}

1. Node $B$ broadcasts "Find" message to find a reverse path to reach the inneighbor $A$. This "Find" message contains source ID (i.e. $B$ here), destination ID (i.e. $A$ here), node power and expiration bit with preloaded value 3 .

2. if some node $C$ receives a "Find" message, then

2.a. if it is the destination node, it records the node power and adds the source ID in its out-neighbor list. Broadcast an "acknowledgement" to the source node.

2.b. end if.

2.c. else if it is not the destination node and the expiration bit is greater than zero, then the node will append its node ID as intermittent ID and rebroadcast the message by reducing the size of expiration bit count by one.

2.d. end else if.

3. in the other case, the broadcasted messages will be dropped.

4. end if. 


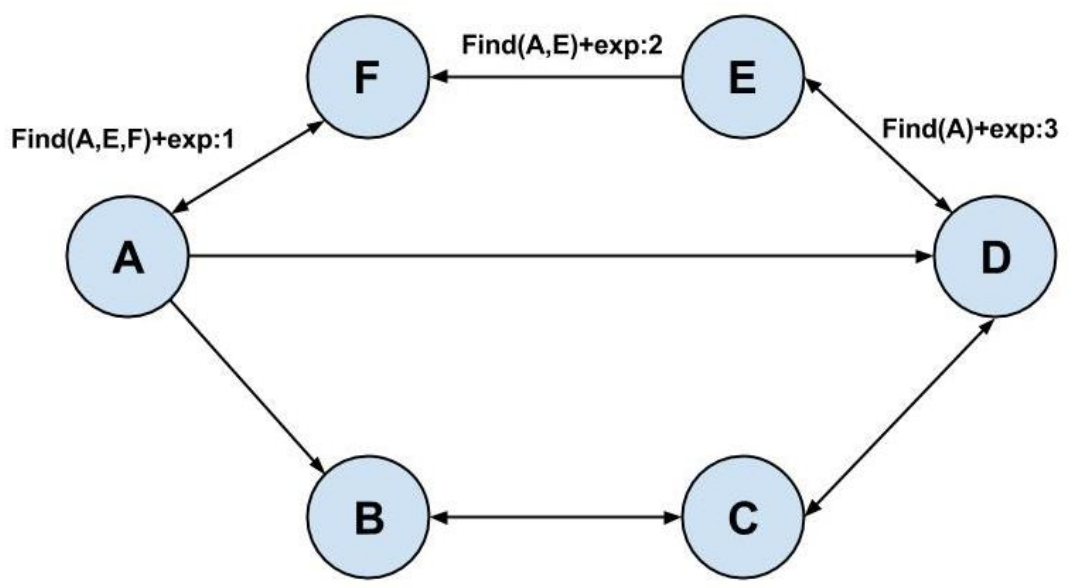

Figure 2. Example of Reverse Path. Here $D \rightarrow E \rightarrow F \rightarrow A$ is Considered as Reverse Path

Let us consider Figure 2 to explain the reverse path identification process in WHSNs. In this network $A, B, C, D, E, F$ are sensors with different transmission ranges. The direction in this connectivity represents their neighbor node relationship. As per Figure 2, $A$ and $F, B$ and $C, D$ and $E$ are in-out neighbors. Let us assume the node $D$ wants to find the destination node $A$. Here $D$ is an in-neighbor to $A$ but $A$ is out-neighbor to $D$. So the node $D$ can receive "request" message from node $A$, but due to its diverse transmission power it will not get "acknowledgement" from node $A$. So node $D$ should find a reverse path to reach node $A$. Source node $D$ will broadcast a "Find" message that contains source ID, destination ID and node power. Expiration bit of "Find" is set to 3. The "find" message will be received and further processed by node $E$ and $C$, because these nodes are having asymmetric links in between them. Each node will check whether the destination ID is their own ID or not. Here this check fails and so this "Find" message will be rebroadcasted by adding the intermittent ID. As per Figure 2, node $C$ can forward the modified "Find" message to node $B$ with an appended intermittent ID. In the same way, node $E$ also rebroadcasts "Find" message to node $F$. Again, node $F$ will rebroadcast the "Find" message and will locate the destination node $A$. Finally, node $A$ will store the incoming node ID in it's out-neighbor list and will store its power. An "acknowledgement" message will be broadcasted to node $D$ from node $A$ afterwards.

\subsection{Selection Part}

The nature of simple wireless communication is broadcasting. So the easiest and reliable way to deliver a packet from source to sink is flooding. However, flooding in a large network will create flooding storm. In order to reduce the network overhead, we adopt unicasting data in a selection part. This selection part comprises of two phases and both are running in a source node: (1) Selection phase and (2) Unicast the data phase. Selection phase means processing the stored data by a source node and so it uses a separate array of memory. It sorts the responded neighbor nodes based on node power. The sample processing data and processed data are shown in Table 1 and 2. This method is explained in Algorithm 3. After selection phase, the source node unicasts the data to its best-hop (A_072). This work will be continued till the message reaches the sink.

Table 1. Sample Value Recorded from a Source before Selection

\begin{tabular}{ccccccc}
\hline $\begin{array}{c}\text { Receiver } \\
\text { ID }\end{array}$ & $\begin{array}{c}\text { Source_Location } \\
\text { (A_018) }\end{array}$ & Receiver_Location & $\begin{array}{c}\text { Node } \\
\text { Power }\end{array}$ & $\begin{array}{c}\text { In-out } \\
\text { list }\end{array}$ & $\begin{array}{c}\text { Out } \\
\text { list }\end{array}$ & $\begin{array}{c}\text { Intermittent } \\
\text { Nodes }\end{array}$ \\
\hline
\end{tabular}




\begin{tabular}{|c|c|c|c|c|c|c|c|c|c|c|}
\hline & $X$ & $\mathrm{Y}$ & $\mathrm{Z}$ & $X$ & $\mathrm{Y}$ & $\mathrm{Z}$ & & & & \\
\hline A_026 & 10.58 & 14.21 & 23.91 & 14.61 & 10.82 & 14.75 & 84.93 & A_026 & NULL & NULL \\
\hline A_021 & 10.58 & 14.21 & 23.91 & 20.65 & 25.35 & 24.57 & 53.91 & NULL & A_021 & A_039 \\
\hline A_072 & 10.58 & 14.21 & 23.91 & 00.34 & 19.24 & 26.28 & 96.74 & NULL & A_072 & A_028 \\
\hline A_028 & 10.58 & 14.21 & 23.91 & 3.51 & 08.10 & 35.62 & 48.46 & A_028 & NULL & NULL \\
\hline A_039 & 10.58 & 14.21 & 23.91 & 09.41 & 15.31 & 12.82 & 83.92 & A_039 & NULL & NULL \\
\hline
\end{tabular}

Table 2. Sample Recorded after Selection

\begin{tabular}{|c|c|c|c|c|c|c|c|c|c|c|}
\hline \multirow{2}{*}{$\begin{array}{l}\text { Receiver } \\
\text { ID }\end{array}$} & \multicolumn{3}{|c|}{$\begin{array}{l}\text { Source_Location } \\
\text { (A_018) }\end{array}$} & \multicolumn{3}{|c|}{ Receiver_Location } & \multirow{2}{*}{$\begin{array}{l}\text { Node } \\
\text { Power }\end{array}$} & \multirow{2}{*}{$\begin{array}{l}\text { In-out } \\
\text { list }\end{array}$} & \multirow{2}{*}{$\begin{array}{l}\text { Out } \\
\text { list }\end{array}$} & \multirow{2}{*}{$\begin{array}{l}\text { Intermittent } \\
\text { Nodes }\end{array}$} \\
\hline & $\mathrm{X}$ & $\mathrm{Y}$ & $\mathrm{Z}$ & $\mathrm{X}$ & $\mathrm{Y}$ & $\mathrm{Z}$ & & & & \\
\hline A_072 & 10.58 & 14.21 & 23.91 & 00.34 & 19.24 & 26.28 & 96.74 & NULL & A_072 & A_028 \\
\hline A_026 & 10.58 & 14.21 & 23.91 & 14.61 & 10.82 & 14.75 & 84.93 & A_026 & NULL & NULL \\
\hline A_039 & 10.58 & 14.21 & 23.91 & 09.41 & 15.31 & 12.82 & 83.92 & A_039 & NULL & NULL \\
\hline A_021 & 10.58 & 14.21 & 23.91 & 20.65 & 25.35 & 24.57 & 53.91 & NULL & A_021 & A_039 \\
\hline A_028 & 10.58 & 14.21 & 23.91 & 3.51 & 08.10 & 35.62 & 48.46 & A_028 & NULL & NULL \\
\hline
\end{tabular}

\section{Algorithm 3: Sorting at Source Node}

1. Input: A Queue list ' $L$ ' contains $\{\{$ Receiver ID, $/ *$ Optional */Intermittent ID $\}+$ Power $\}$

2. Pre-condition: An unsorted queue list ' $\mathrm{L}$ '

3. Loop Invariant: Identify $\mathrm{MAX}=\{\{$ Receiver ID, $/ *$ Optional $* /$ Intermittent ID ++ Power $\}$

4. Assume: i, j, n, MIN: float variables.

5. Calculate $\mathrm{n}=$ Number of elements in Queue list ' $\mathrm{L}$ '

6. $\quad$ for $(\mathrm{j}=0 ; \mathrm{j}<\mathrm{n} ; \mathrm{j}++)\{$

7. $\mathrm{MIN}=\mathrm{j}$;

8. for $(\mathrm{i}=\mathrm{j}+1, \mathrm{i}<\mathrm{n} ; \mathrm{i}++)\{$

9. if(Queue[i] < Queue[MIN]) \{

10. $\mathrm{MIN}=\mathrm{i} ;\}$ \}

11. If(MIN!=j)

12. $\{\operatorname{swap}($ Queue$[\mathrm{j}]$, Queue[MIN] $\}$

13. Select: Best-hop $=$ Queue[n]

14. Post-condition: A list ' $L$ ' contains sorted $\{\{$ Power $\}$, Receiver ID/* Optional */Intermittent ID\}

15. End algorithm.

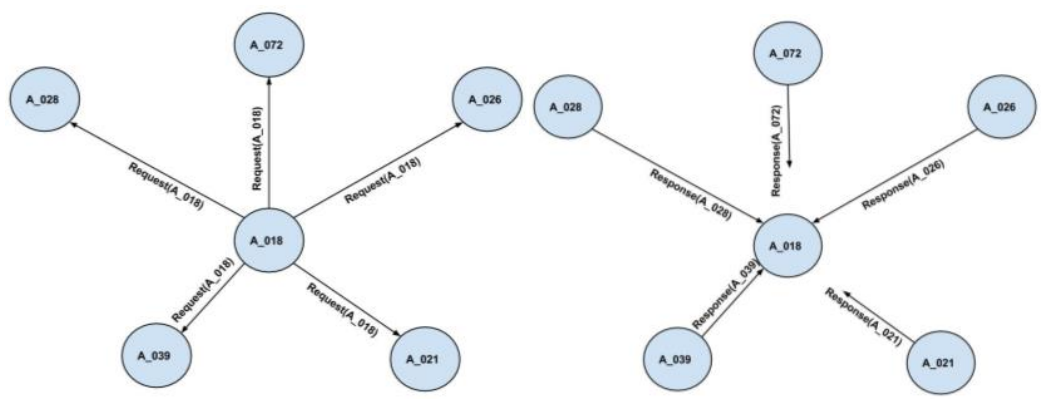

(a)

(b) 


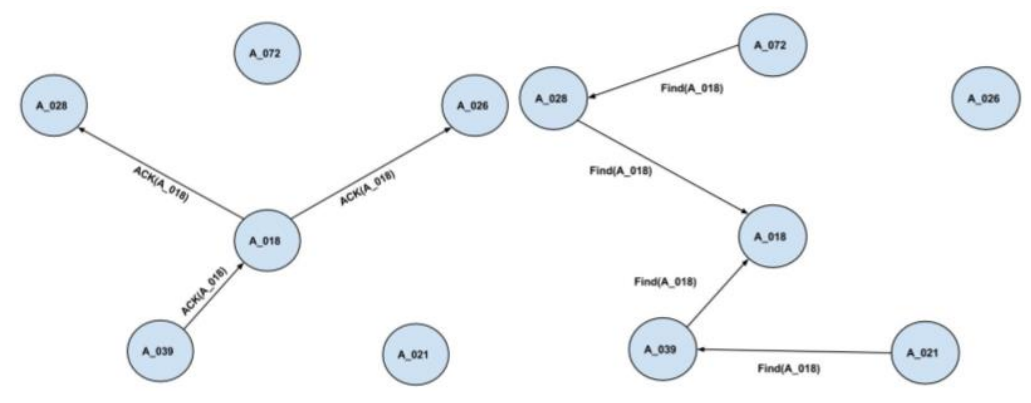

(c)
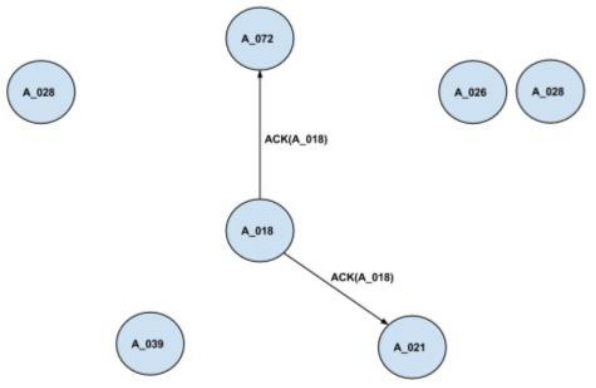

(e)

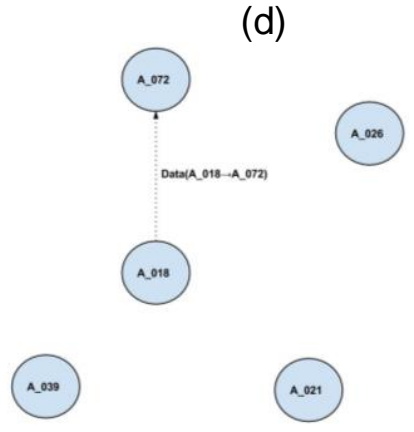

(f)

Figure 3. The Whole Representation of Our Proposed Work

\section{Simulation Results of Our Proposed Protocol}

In this section, we present simulation results for our work based on simplified MAC, without considering packet losses, the Euclidean model with minimum or no greedy failures and random node deployment.

\subsection{Simulation Settings}

We have implemented a simulation package based on NS-2 [37] which is one of the open-source tools available freely. In all the simulations, 500 sensor nodes are randomly deployed in $5000 \times 1500 \mathrm{~m}^{2}$ test bed. 20 sensor nodes are randomly selected as source nodes. Each sensor node has different transmission ranges. The sink is placed at the center of the test bed and assigned an infinite power supply. The request message length is 20 bytes and the response message length is 25 bytes. The total size of find message is 25 bytes. The data transmission rates of nodes are in the range of $250 \mathrm{kbps}$ and are disseminated in ISM band. The minimum and maximum transmission ranges for an individual node is $5 \mathrm{~m}$ and $25 \mathrm{~m}$ respectively. One single source node can generate one packet per second. Packet size is 80 bytes, and the overall simulation setup time is 50 minutes. We use first order radio model to compute the energy consumption of all the nodes. The parameter values used in the simulations are presented in Table 3. The basic settings are common to all the experiments.

Table 3. Simulation Settings

\begin{tabular}{ll}
\hline Network Area & $5000 \mathrm{~m} \times 1500 \mathrm{~m}$ \\
\hline Total Sensor Nodes & 500 \\
\hline Data Rate at MAC layer & $250 \mathrm{kbps}$ \\
\hline Topology Configuration & Randomized \\
\hline Packet loss rate & $0 \%$ \\
\hline
\end{tabular}




\begin{tabular}{ll}
\hline Node failure rate & $0 \%$ \\
\hline Transmission Range & $5 \mathrm{~m}$ to $25 \mathrm{~m}$ \\
\hline Overall Simulation Time & 50 minutes \\
\hline
\end{tabular}

Three different scenarios are designed to evaluate the performance of the proposed work.

- Varying active nodes scenario: Here we introduced a new method that sensor nodes are in either active or inactive state. Every sensor node cannot be assumed as active in a Euclidean plane throughout the simulation cycle.

- Random walk scenario: Every sensor node moves in a Euclidean plane according to Random Walk Mobility Model [38]. A sensor can select its own new position by choosing its speed and direction from the range [minimum speed: 0, maximum speed: $2 \pi$ ]. Every node movement continues for an interval time of 10 seconds. New speed and direction can be taken at the end of each interval time.

- Random sleep wake up scenario: A Random Independent Sleeping (RIS) scheme proposed in [39] is employed in our work to extend the network lifetime. This RIS scheme divides the entire simulation time into $\zeta_{\text {sleep }}$ intervals. At the beginning of each interval, each node works actively with probability value $\rho$ and sleeps with a probability $1-\rho$. This sleep and wake up cycle is decided by $\rho$.

For a fair performance analysis, in addition to our proposed protocol, we have implemented one more probabilistic routing scheme for a heterogeneous sensor network: ProHet [33]. ProHet uses beacon messages to identify its hop-nodes. It pushes the sink to produce acknowledgement messages, when a message reaches sink. The primary MAC protocol is IEEE 802.11, and the configuration of the MAC protocol is described as follows: For beacon-based ProHet, the request/response handshake function is turned off to decrease communication overhead since it is not necessary for this scheme. The beacon message is set to 20 bytes. For the energy model described in the preliminaries, the energy spent by transmitter source on transmitting or receiving 1-bit data (i.e., $x_{11}$ and $x_{12}$ ) is set to $50 \mathrm{~nJ} / \mathrm{bit}$, the transmitting amplifier $\left(\mathrm{x}_{2}\right)$ is set to $10 \mathrm{pJ} / \mathrm{bit} / \mathrm{m}^{2}$, the energy spent by an intermittent node is $0.5 \mathrm{~nJ} / \mathrm{bit}$ and the propagation loss exponent $(\mathrm{k})$ is set to 2 . In each simulation, 20 nodes are selected as source nodes and each source node generates 40 data packets with a payload of 128 bytes. The simulation does not end until the sink receives all the data packets generated in the network and the simulation results are the average of 50 autonomous runs.

\subsection{Varying Active Nodes Scenario}

In this simulation, all sensor nodes are able to send and receive beacon or request/response messages. We first analyze the delivery ratio of both the protocols. As can be seen from Figure 4 (a), the delivery ratio of our proposed protocol is better than ProHet. The reason is ProHet struggles to make its forwarding decision because of low number of active nodes. When the number of active nodes increased, the delivery ratio of both the protocols is increased. When the numbers of active nodes are greater than 350 , both protocols are getting same delivery ratio.

Latency of the proposed protocol is analyzed in terms of seconds and shown in Figure 4 (b). It shows that latency is inversely connected with number of active nodes. ProHet gives lower latency than our proposed protocol. There are two reasons for this better performance. First, ProHet does not consider the power factor on each node. It uses simple beacon signals to select two best hops. So the process of forwarder node selection becomes simple and saves more time. Second is, the source node gets two-hop receivers easily when the active nodes are increased. In contrast, our proposed protocol selects the best-hop based on node power. Thus it causes few delays in each source node, and reflects in the analysis. 


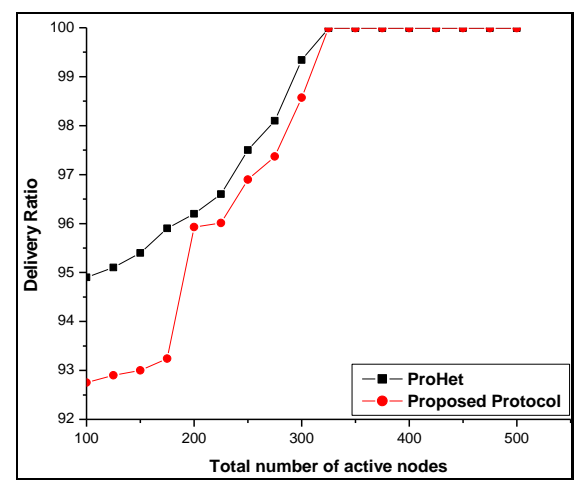

(a)

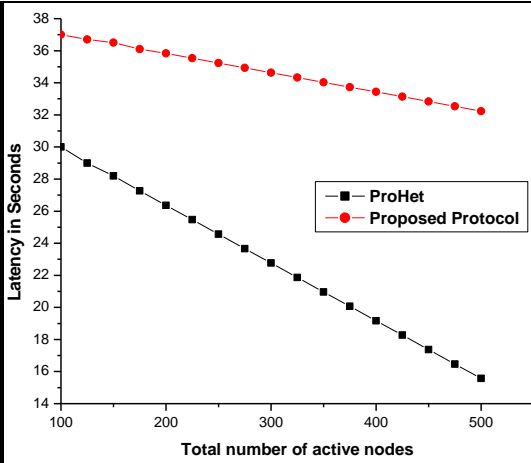

(b)

Figure 4. Delivery Ratio and Latency of Proposed Protocol Against ProHet in Varying Active Nodes Scenario

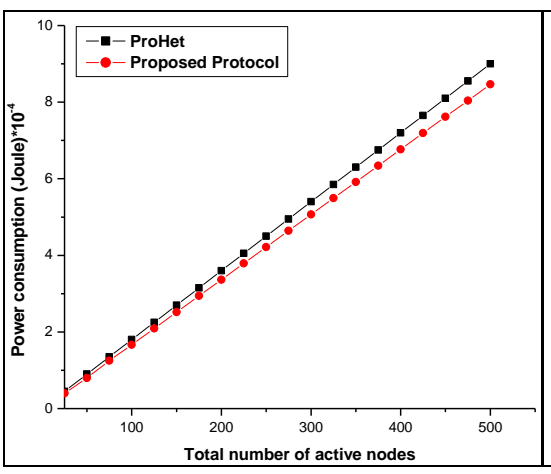

(a)

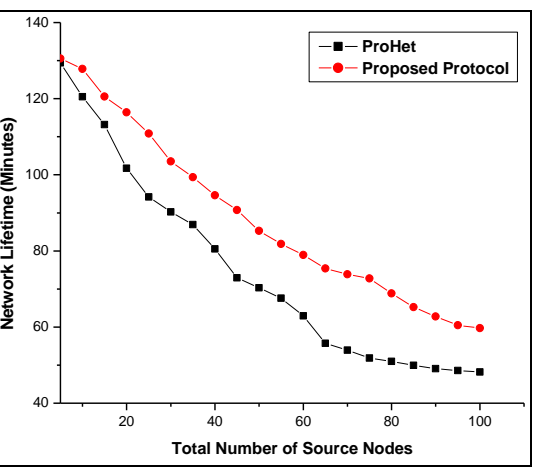

(b)

Figure 5. Energy Consumption and Network Lifetime of Both the Protocols

Figure5 (a) shows the energy consumption which is the sum of the energy spent by each node in the overall network. Here our proposed protocol and ProHet are much robust because they decide their forwarding decision based on the deployed network topology. When the number of active node increases, the energy consumption of proposed protocol and ProHet increases slightly due to the sub-optimal energy routes. Comparatively, our proposed protocol gives a better performance at the end and consumes less power than ProHet. ProHet loses more energy while forwarding the same data to two-hops. These two-way receivers again choose two other best-hops. That is a major cause of power consumption. Our proposed protocol chooses a single best-hop rather than choosing two best-hops and saves more power. It also reflects in network lifetime, shown in Figure 5 (b). Our proposed protocol prolongs the network lifetime by choosing a maximum powered node. It also follows one-hop receiver model and thus saves more energy than ProHet.

\subsection{Performance of Proposed Protocol in Random Walk Scenario}

In this simulation, we evaluate the performance of our proposed protocol in random walk scenarios in which the network topology changes frequently due to node mobility. The parameters of the Random Walk Mobility Model are set as follows: minspeed is set to $0 \mathrm{~m} / \mathrm{s}$, and maxspeed is varied from 0 to $50 \mathrm{~m} / \mathrm{s}$ to provide different levels of mobility.

Figure 6 (a) shows the delivery ratio of both the protocols under random walk. When all the nodes are in fixed state, both the protocols are providing most robust data delivery. When the node movement reaches $12.5 \mathrm{~m} / \mathrm{s}$, deviation in packet delivery occurs. This 
deviation becomes vital consideration in our analysis. ProHet struggles internally to make two-way receiver model because of node mobility. Our proposed protocol comparatively gives better performance than ProHet, because of single-hop selection. The same reason causes more latency in ProHet than our proposed protocol. Based on collected information, our proposed protocol forwards the data. But ProHet waits until the node probability threshold meets. So ProHet shows more latency than our proposed protocol.

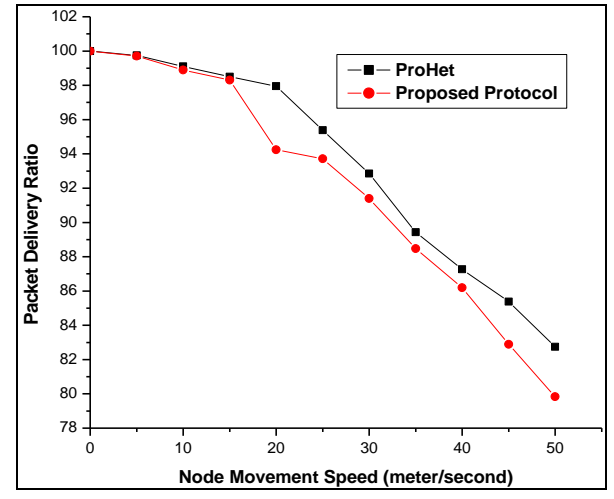

(a)

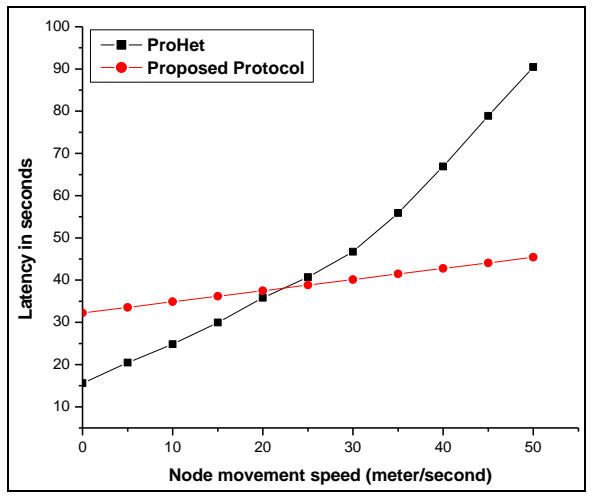

(b)

Figure 6. Delivery Ratio and Latency of Proposed Protocol Against ProHet in Random Walk Scenario

When the node movement speed increases, both the protocols are spending more energy to find its best-hop. Due to mobility, only few nodes get qualified in the hop-node contention process. In this preliminary circumstance, our proposed protocol consumes less power than ProHet. Both beacons and request messages are easily get outdated when the node movement speed increases. When node movement speed exceeds $25 \mathrm{~m} / \mathrm{s}$, source nodes in both the protocols are unable to gather enough forwarder information accurately. More closely looking, this node movement speed behaves like a saturated point, because both the protocols are consuming moreover same energy afterwards. This reflects the effect in network lifetime shown in Figure 6 (b).

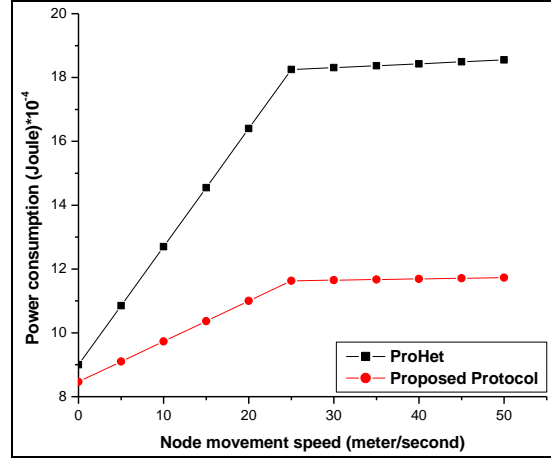

(a)

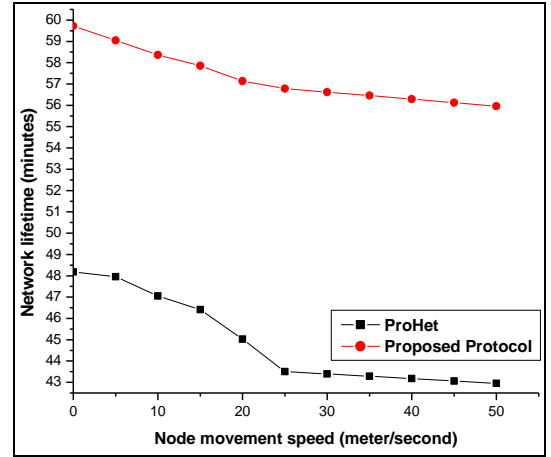

(b)

Figure 7. Energy Consumption and Network Lifetime of Both the Protocols under Random Walk Scenario 


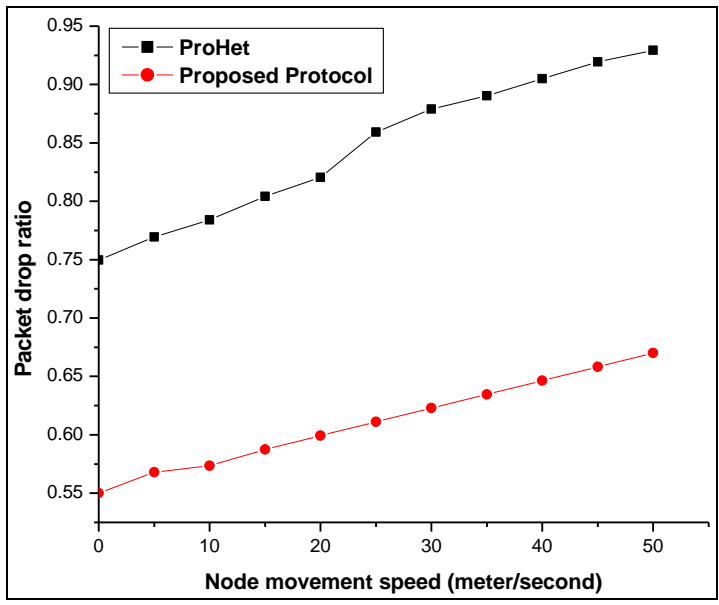

\section{Figure 8. Packet Drop Ratio of Both the Protocols under Random Walk Scenario}

Figure 8 shows the total number of packets dropped when all the nodes are in moving state. More number of packet drops occurs in both the protocols when the speed increases. Due to two-way receiver model, the packet drop ratio in ProHet is heavily more than our proposed protocol. Sink also drops the duplicated packets received from any one of source node in two-way receiver model.

\subsection{Performance of Proposed Protocol in Random Sleep Scenario}

In order to measure the performance of simulated protocols in random sleep wake up cycle, RIS scheme $\left(T_{\text {shift }}\right)$ is integrated. In order to integrate RIS scheme in both the protocols, each node broadcasts a beacon or request message only when it shifts between sleep and active state. Neighbor node will be active in the selection process if its remaining active time is large enough to complete forwarding a data packet.

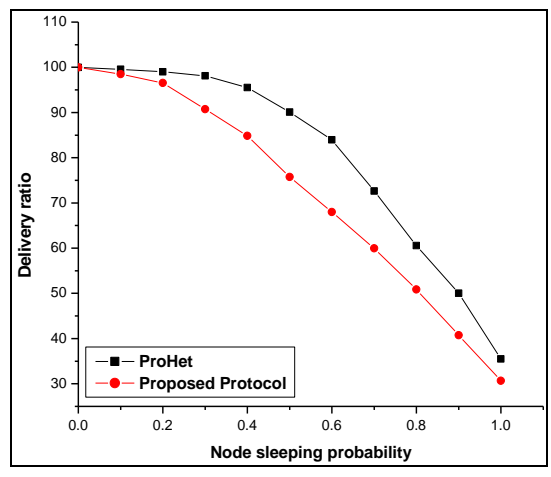

(a)

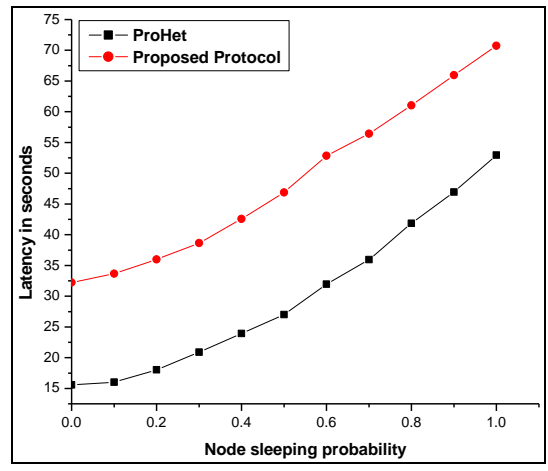

(b)

\section{Figure 9. Delivery Ratio and Latency of Proposed Protocol Against ProHet in Random Sleep Scenario}

From Figure 9 (a) we can conclude that, packet delivery ratio is inversely connected with node sleeping probability. ProHet shows low amount of data delivery at higher sleep probability than our proposed protocol. The main reason is, more number of nodes is sleep, when source node broadcasts beacons. Thus it failed to make probability threshold. Reverse path acknowledgement makes more latency in our proposed protocol. Individual delay based responses are eliminating congestion mostly in our work, but it creates more end-to-end delay. It is shown in Figure 9 (b). 


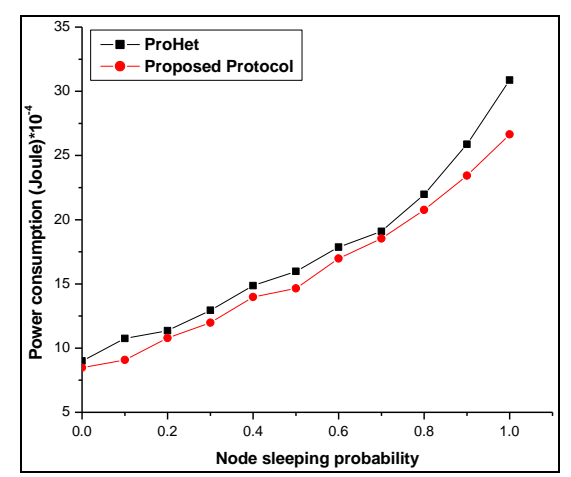

(a)

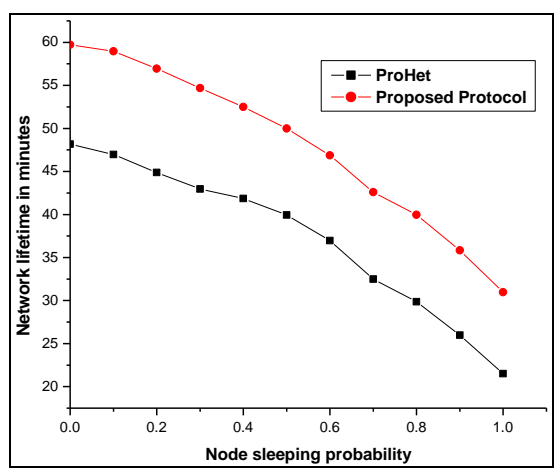

(b)

Figure 10. Energy Consumption and Network Lifetime of Both the Protocols under Random Sleep Scenario

Energy consumption of our proposed protocol is better than ProHet during node sleeping scenario. Whenever the node sleeping probability is more than 50\%, the remaining active nodes are working more to complete the data transmission process. Moreover ProHet struggles in local minimum problem. Source node couldn't find best two-hop receivers other than the source itself. In order to recover from local minimum, ProHet selects unsuitable nodes and protest them to work as hop-nodes. It reduces the entire network lifetime.

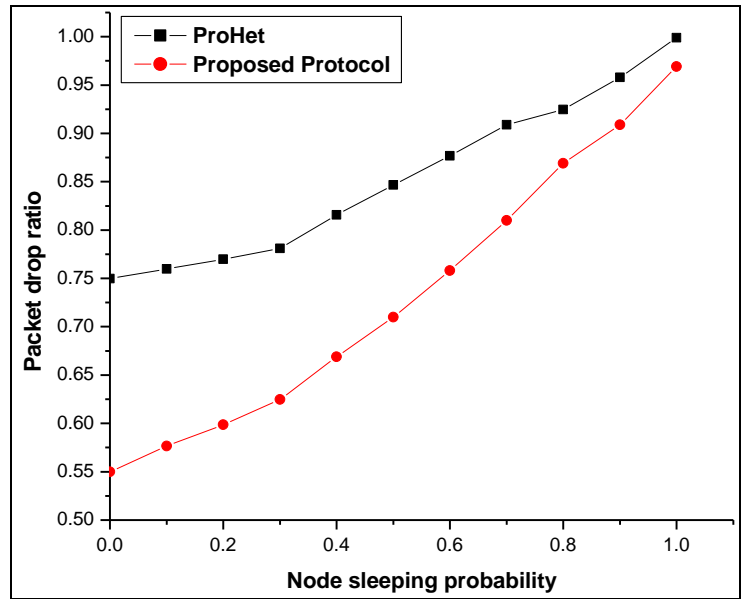

Figure 11. Packet Drop Ratio of Both the Protocols under Random Sleep Scenario

Figure 11 shows the total number of packets dropped when all the nodes are under random sleep wake-up state. More number of packet drops occurs in both the protocols when the node sleeping probability increases. Due to two-way receiver option and lack of availability of best-hops, the packet drop ratio in ProHet is heavily more than our proposed protocol.

\section{Conclusion and Future Work}

Power aware routing is a hot research aspect in WSNs. In this work, we address the problem of asymmetric links in WHSNs and propose a novel power aware routing protocol which provides energy efficient, loop-free, stateless sensor-to-sink routing in highly unstable asymmetric scenarios. The performance of our proposed protocol is 
evaluated under different cases. Simulation results show that our protocol outperforms well in most scenarios and also consumes less power than the other protocol based on collected neighborhood information in highly dynamic scenarios.

Congestion control is mainly achieved by delay scheme, which is a major contribution in this work. But if we look closer, we can understand that the energy conservation of hop-nodes is high because of delay based individual reply. Our future work is that, if some improvement is adopted in this delay based reply system, then it would be much more efficient.

\section{References}

[1] F. Akyildiz, W. Su, Y. Sankarasubramaian, and E. Cayirci, "A Survey on Sensor Networks," IEEE Communication Magazine, vol. 40 no. 8, (2002), pp. 102-114.

[2] I. A. Essa, "Ubiquitous Sensing for Smart and Aware Environments," IEEE Personal Communications, vol. 7, (2000), pp. 47-49.

[3] A. M. Mainwaring, D. E. Culler, J. Polastre, R. Szewczyk, and J. Anderson, "Wireless Sensor Networks for Habitat Monitoring," Proceedings of ACM International Workshop on Wireless Sensor Network Applications, (2002).

[4] D. A. Patterson, "Rescuing our families, our neighbors and ourselves," ACM Communications, vol. 48 no. 11 , (2005), pp. $29-31$.

[5] C. Intanagonwiwat, R. Govindan, and D. Estrin. D, "Directed Diffusion: a scalable and robust communication paradigm for sensor networks," Proceedings of International Conference on Mobile Communication Networks, (2000), pp. $56-67$.

[6] A. Manjeshware, and D. P. Agarwal, "TEEN: a routing protocol for enhanced efficiency in wireless sensor networks," Proceedings of International Conference on Parallel Distributed Process, (2001), pp. $2009-2015$.

[7] F. Ye, H. Y. Luo, J. Cheng, S. W. Lu, and L. X. Zhang, "A two-tier data dissemination model for large-scale wireless sensor networks," Proceedings of International Conference on Mobile Computing Networks, (2002), pp. 1-4.

[8] V. Ramasubramanian, and D. Mosse, "BRA: a bidirectional routing abstraction for asymmetric mobile ad hoc networks," IEEE/ACM Transactions on Networks, vol. 16 no. 1, (2008), pp. 116-129.

[9] S. Ben Alla, A. Ezzati, Beni Hasane, and M. L. Hasnaoui, "Hierarchal adaptive balanced energy efficient routing protocol," Proceedings of International Conference on Multimedia Computer Systems, (2011).

[10] M. Perillo, M. Cheng, and W. Heinzelman, "An analysis of strategies for mitigating the sensor network hot spot problem," Proceedings of International Conference on Mobi Quitos, (2005), pp. 474478.

[11] H. Rivas, T. Voigt, and A. Dunkels, "A simple and efficient method to mitigate the hot spot problem in wireless sensor networks," Proceedings of International Conference on Performance Control Wireless Sensor Networks, (2006).

[12] W. Y. Zhang, X. J. Du, J. Wu, S. D. Soysa, and Y. Liu, "Near-minimum energy routing in heterogeneous sensor networks," Proceedings of International Conference on IEEE GLOBECOM, (2010), pp. 1-5.

[13] I. Stojmenovic, and X. Lin, "Power-Aware Localized Routing in Wireless Networks," IEEE Transactions on Parallel and Distributed Systems, vol. 12 no. 11, (2001), pp. 1122-1133.

[14] K. Kalpakis, K. Dasgupta, and P. Namjoshi, "Efficient Algorithms for Maximum Lifetime Data Gathering and Aggregation in Wireless Sensor Networks," Computer Networks, vol. 42, (2003), pp. 697-716.

[15] Haibo Zhang and Hong Shen, "Energy-efficient beaconless Geographic Routing in Wireless Sensor Networks," IEEE Transactions on Parallel and Distributed Systems, vol. 21, no. 6, (2010), pp. 881-896.

[16] H. Takagi, and L. Kleinrock, "Optimal Transmission Ranges for Randomly Distributed Packet Radio Terminals," IEEE Transactions on Communications, vol. 32 no. 3, (1984), pp. $246-257$.

[17] S. Wu, and K. S. Candan, "GPER: Geographic Power Efficient Routing in Sensor Networks," Proceedings of IEEE International Conference in Network Protocols, (2004), pp. 161-172.

[18] J. Kuruvila, A. Nayak, and I. Stojmenovic, "Hop Count Optimal Position Based Packet Routing Algorithms for Ad Hoc Wireless Networks with a Realistic Physical Layer," Proceedings of First IEEE International Conference on Mobile Ad-Hoc and Sensor Systems, (2004), pp. 398 - 405.

[19] M. Heissenbuttel, T. Braun, T. Bernoulli, and M. Walchli, "BLR: Beacon-Less Routing Algorithm for Mobile Ad Hoc Networks," Computer Communications, vol. 11, (2004), pp. 1076-1086.

[20] H. Fußler, J. Widmer, M. Kasemann, M. Mauve, and H. Hartenstein, "Contention-Based Forwarding for Mobile Ad Hoc Networks," Ad Hoc Networks, vol. 1, (2003), pp. 351-369.

[21] B. Blum, T. He, S. Son, and J. Stankovic, "IGF: A State-Free Robust Communication Protocol for Wireless Sensor Networks,” Technical Report CS-2003-11, Univ. of Virginia. (2003). 
[22] I. S. P. Bose, P. Morin, and J. Urrutia, "Routing with Guaranteed Delivery in Ad Hoc Wireless Networks," Proceedings of Third ACM International Workshop Discrete Algorithms and Methods for Mobile Computing and Communications, (1999), pp. 48-55.

[23] H. T. K. B. Karp, "GPSR: Greedy Perimeter Stateless Routing for Wireless Networks," Proceedings of ACM MobiCom, (2000), pp. 243-254.

[24] F. Kuhn, R. Wattenhofer, and A. Zollinger, "Worst-Case Optimal and Average-Case Efficient Geometric Ad-Hoc Routing," Proceedings of ACM MobiCom, (2003), pp. 267-278.

[25] L. Barriere, P. Fraigniaud, L. Narayanan, and J. Opatrny, "Robust Position-Based Routing in Wireless Ad Hoc Networks with Irregular Transmission Ranges," Wireless Communications and Mobile Computing, vol. 3, (2003), pp. 141-153.

[26] J. A. Karaki, and A. Kamal, "Routing techniques in Wireless Sensor Networks: a survey," IEEE Transactions on Wireless Communications, vol.11, no. 6, (2004), pp. 6-28.

[27] H. Frey, and I. Stojmenovic, "On Delivery Guarantees of Face and Combined Greedy-Face Routing in Ad Hoc and Sensor Networks," Proceedings of ACM MobiCom, (2006), pp. 390-401.

[28] B. Elbhiri, R. Saadane, and D. Aboutajdine, "Stochastic distributed energy-efficient clustering for heterogeneous wireless sensor networks," ICGST International Journal Computers Networks and Internet Research, vol. 9, no. 2, (2009), pp. $11-17$.

[29] X. Chen, W. Y. Qu, H. L. Ma, and K. Q. Li, "A geography-based heterogeneous hierarchy routing protocol for wireless sensor networks," IEEE International Conference on High Performance Computing and Communications, (2008), pp. $767-774$.

[30] X. Du, and F. Lin, "Designing efficient routing protocol for heterogeneous sensor networks," IEEE International Conference on Computers Communications, (2005), pp. 51-58.

[31] A. Behzadan, A. Anpalagan, and B. Ma, "Prolong network lifetime via nodal energy balancing in wireless heterogeneous sensor networks," Proceedings of IEEE ICC, (2011), pp. $1-5$.

[32] D. L. Guidoni, A. Boukerche, L. A. Villas, F. S. H. Souza, R. A. H. Mini, and A. A. F. Loureiro, "A framework based on small world features to design HSNS topologies with QoS," IEEE Symposium on Computers and Communications, (2010).

[33] Xiao C., Zanxun D., Wenzhong L., Yuefei H., Jie W., Hongchi S., and Sanglu L., "ProHet: A probabilistic routing protocol with assured delivery rate in wireless heterogeneous sensor networks," IEEE Transactions on wireless communications, vol. 12, no. 4, (2013), pp. $1524-1531$.

[34] Y. F. Hu, W. Z. Li, X. Chen, S. L. Lu, and J. Wu, “A probabilistic routing protocol for heterogeneous sensor networks," IEEE International Conference on Networking Architecture Storage, (2010).

[35] W. R. Heinzelman, A. Chandrakasan, and H. Balakrishnan, "Energy-Efficient Communication Protocol for Wireless Micro-sensor Networks," Proceedings of $33^{\text {rd }}$ Hawaii International Conference on System Sciences, (2000), pp. 4-7.

[36] M. D. Yarvis, N. Kushalnagar, H. Singh, A. Rangarajan, Y. Liu, and S. Singh, "Exploiting heterogeneity in wireless sensor networks," IEEE INFOCOM, (2005), pp. 878 - 890.

[37] http://www.isi.edu/nsnam/ns/

[38] M. Sanchez, and P. Manzoni, "A Java-Based Ad Hoc Networks Simulator," Proceedings of SCS Western Multi-conference on Web-Based Simulation Track, (1999).

[39] S. Kumar, T. H. Lai, and J. Balogh, "On K-coverage in a Mostly Sleeping Sensor Network," Proceedings of ACM MobiCom, (2004), pp. $144-158$.

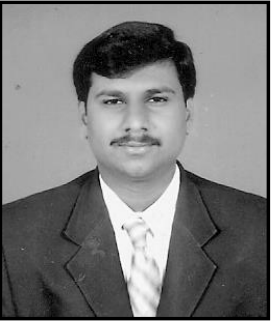

M. Viju Prakash, he is 31 years old. He completed a B.E. and M.E. degree in computer science and engineering from Anna University, Chennai, India. $\mathrm{He}$ is currently working as an assistant professor in the Department of Computer Science and Engineering, St. Xavier's Catholic College of Engineering, Nagercoil, India. He is also pursuing his Ph.D. degree in the Department of Computer Science and Engineering, Manonmaniam Sundaranar University, Tirunelveli, India. His areas of interest are wireless sensor networks, secure routing techniques, congestion control and mobile ad hoc networks. 


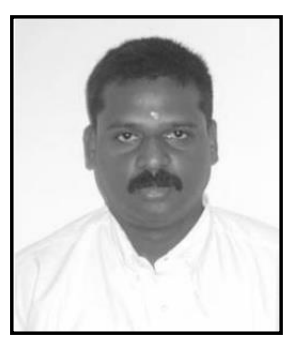

B. Paramasivan, he is 46 years old. He completed a B.E. degree in computer science and engineering from Madurai Kamaraj University, Tamil Nadu, India. He obtained an M.E degree in computer science and engineering from Jadavpur University, Kolkatta, India. He received Ph.D. degree from Anna University, Chennai, India in wireless sensor networks. He is a Professor and Head of the Department of Computer Science and Engineering, National Engineering College, Kovilpatti, Tamil $\mathrm{Nadu}$, India. He has published more than fifteen papers in national and international journals. He has also presented more than thirty papers in various national and international conferences. He has organized more than ten seminars sponsored by various government funding agencies. He is an active member of various professional bodies such as IE and CSI. His research interests include wireless ad hoc and sensor networks and highperformance networking. 